\title{
Challenges in Inflationary Magnetogenesis: Constraints from Strong Coupling, Backreaction and the Schwinger Effect
}

\author{
Ramkishor Sharma ${ }^{1},{ }^{*}$ Sandhya Jagannathan ${ }^{1},{ }^{\dagger}$ T. R.Seshadri ${ }^{1}, \ddagger$ and Kandaswamy Subramanian ${ }^{2 \S}$ \\ ${ }^{1}$ Department of Physics 83 Astrophysics, University of Delhi, New Delhi-110007 India. and \\ ${ }^{2}$ IUCAA, Post Bag 4, Pune University Campus, Ganeshkhind, Pune-411007 India.
}

\begin{abstract}
Models of inflationary magnetogenesis with a coupling to the electromagnetic action of the form $f^{2} F_{\mu \nu} F^{\mu \nu}$, are known to suffer from several problems. These include the strong coupling problem, the back reaction problem and also strong constraints due to Schwinger effect. We propose a model which resolves all these issues. In our model, the coupling function, $f$, grows during inflation and transits to a decaying phase post inflation. This evolutionary behaviour is chosen so as to avoid the problem of strong coupling. By assuming a suitable power law form of the coupling function, we can also neglect back reaction effects during inflation. To avoid back reaction post-inflation, we find that the reheating temperature is restricted to be below $\approx 1.7 \times 10^{4} \mathrm{GeV}$. The magnetic energy spectrum is predicted to be non-helical and generically blue. The estimated present day magnetic field strength and the corresponding coherence length taking reheating at the QCD epoch $(150 \mathrm{MeV})$ are $1.4 \times 10^{-12}$ $\mathrm{G}$ and $6.1 \times 10^{-4} \mathrm{Mpc}$, respectively. This is obtained after taking account of nonlinear processing over and above the flux freezing evolution after reheating. If we consider also the possibility of a non-helical inverse transfer, as indicated in direct numerical simulations, the coherence length and the magnetic field strength are even larger. In all cases mentioned above, the magnetic fields generated in our models satisfy the $\gamma$-ray bound below a certain reheating temperature.
\end{abstract}

\section{INTRODUCTION}

Magnetic fields have been observed over a wide range of length scales in the universe. They have been detected in galaxies, galaxy clusters and even in intergalactic voids [1-4]. Gamma ray observations have put a lower bound on the intergalactic magnetic fields of the order $10^{-15} \mathrm{G}$ for fields coherent on Mpc scales [4]. The mechanism responsible for the origin of these fields is not yet clearly understood although considerable work has been done in this regard. There are two approaches in this context. One approach attributes the origin of seed fields to astrophysical batteries which are then further amplified by flux freezing and dynamo action [5-8]. The other approach proposes a primordial origin of seed fields. The proponents of this scenario suggest that the generation of seed fields can possibly occur due to processes in early universe during inflation [9-15], electroweak $[16,17]$ or QCD phase transitions $[18,19]$ (for reviews, see [20-24]). Primordial origin would be more favoured if the observations of magnetic fields in voids is firmed up.

Inflation offers a natural setting to explain observed large scale magnetic fields since large scale features emerge naturally from the theory. However, the magnetic field strength generated during inflation decreases with the expansion factor $a(t)$, very rapidly as $B \propto 1 / a^{2}$, as the standard EM action is conformally invariant. This results in a very low present day strength which is far below the value required to even seed the dynamo. Hence conformal invariance needs to be broken to obtain fields

\footnotetext{
*rsharma@physics.du.ac.in, sharmaram.du@gmail.com

† sandhya0892@gmail.com

¥trs@physics.du.ac.in

$\S$ kandu@iucaa.in
}

of sufficient strengths that are observed today. Inflation provides many scenarios where this is possible, one of them is in which the inflaton field couples to the kinetic term of the electromagnetic (EM) field [10]. However this model is also beset with several problems such as the strong coupling and the back reaction problem. Strong coupling problem occurs if the effective electric charge is high during some epoch of inflation thereby making the perturbative calculations of the EM test field untrustworthy. We will address this issue in more detail in Section V. In some cases, during inflation, the electric and magnetic energy density can overshoot the background energy density. This can end inflation as well as suppress the production of magnetic fields. This is known as the back reaction problem. Models of low energy scale inflation have been suggested where the back reaction problem has been tackled [25]. In some of these models, however, Schwinger effect constraint poses a problem [26]. Schwinger mechanism is the production of charged particles due to electric fields. If the electric field is high enough during inflation, it can generate charged particles. This can lead to the conductivity becoming very high. This will affect the EM field and can result in a low strength of the magnetic field today.

In this paper, we propose a model to tackle all the above problems besieging inflationary magnetogenesis. In our model, we allow the coupling function (function of the inflaton which breaks conformal invariance, $f$ ) to evolve during inflation and also during a matter dominated era from the end of inflation to reheating. By demanding that EM field energy density does not back react on the background even after inflation, we get a constraint on the scale of inflation and the reheating temperature. We give several scenarios which satisfy all the three constraints. They all imply a low reheating scale and a blue spectra for the generated fields, with 
a sub-horizon coherence length. Therefore one has to consider the nonlinear effects discussed by Banerjee and Jedamzik [27] during the radiation dominated era after reheating. The field strength decays while the coherence length increases due to this evolution. The generated field strengths and coherence scales in several of our models are consistent with the potential lower limits from $\gamma$-ray observations.

The outline of the paper is as follows: In Section II and III, we provide a general background about the evolution of EM fields during inflation and further state the resultant form of power spectra of magnetic and electric energy density. In Section IV, we discuss the possible constraints on magnetogenesis arising out of Schwinger effect and show how these can be satisfied. In Section $\mathrm{V}$, we address the issue of strong coupling and give a detailed account of the model which solves this problem. Section VI gives the predictions arising out of our model, without taking nonlinear effects into consideration. In Section VII, we add nonlinear effects into our model, also discussing the mechanism of inverse transfer briefly in this context. Further in Section VIII, we discuss whether our results conform with the constraints obtained from gamma ray observations. Our conclusions are given in Section IX.

\section{EVOLUTION OF ELECTROMAGNETIC FIELD DURING INFLATION}

The standard Maxwell action is invariant under conformal transformation [28] and FRW metric is conformally flat. Due to this the electromagnetic (EM) fluctuations decay rapidly as the square of the scale factor. Hence, breaking of conformal invariance is necessary for inflationary magnetogenesis [9]. We start with the action for the EM field in which the conformal invariance is explicitly broken by introducing a time dependent function $f^{2}(\phi)$, where $\phi$ is the inflaton field, coupled to the kinetic term $\left(F^{\mu \nu} F_{\mu \nu}\right)$ in the action [10].

$$
\begin{aligned}
S & =-\int \sqrt{-g} d^{4} x\left[f^{2}(\phi) \frac{1}{16 \pi} F_{\mu \nu} F^{\mu \nu}+j^{\mu} A_{\mu}\right] \\
& -\int \sqrt{-g} d^{4} x\left[\frac{1}{2} \partial^{\nu} \phi \partial_{\nu} \phi+V(\phi)\right] .
\end{aligned}
$$

Here $F_{\mu \nu}=\partial_{\mu} A_{\nu}-\partial_{\nu} A_{\mu}$, where $A_{\mu}$ is the EM 4potential. The term $j^{\mu} A_{\mu}$ represents the interaction where $j^{\mu}$ is the four current density. The second term in the action incorporates the evolution of the inflaton field. In this paper we have adopted Greek indices $\mu, \nu \ldots$. to represent space-time coordinates and Roman indices $i, j, k \ldots$ to represent spatial coordinates. We follow the metric convention $g_{\mu \nu}=\operatorname{diag}(-,+,+,+)$. To begin with, we neglect the interaction term and assume that there are no free charges. Varying the action with respect to the EM 4-potential, we obtain the following modified form of Maxwell's equations.

$$
\left[f^{2} F^{\mu \nu}\right]_{; \nu}=\frac{1}{\sqrt{-g}} \frac{\partial}{\partial x^{\nu}}\left[\sqrt{-g} g^{\mu \alpha} g^{\nu \beta} f^{2}(\phi) F_{\alpha \beta}\right]=0 .
$$

Varying the action with respect to the scalar field we obtain the following equation.

$$
\frac{1}{\sqrt{-g}} \frac{\partial}{\partial x^{\nu}}\left[\sqrt{-g} g^{\mu \nu} \partial_{\mu} \phi\right]-\frac{d V}{d \phi}=\frac{f}{8 \pi} \frac{d f}{d \phi} F_{\mu \nu} F^{\mu \nu} .
$$

Here EM field is assumed to be a test field and hence, it will not affect the evolution of the background which is dominated by the scalar field potential during inflation. The scalar field $\phi$ is assumed to be homogeneous, having only time dependence. Adhering to the homogeneity and isotropy of the universe, we work in FRW space-time and further assume it to be spatially flat,

$$
\begin{aligned}
d s^{2} & =-d t^{2}+a^{2}(t)\left[d x^{2}+d y^{2}+d z^{2}\right] \\
& =a^{2}(\eta)\left[-d \eta^{2}+d x^{2}+d y^{2}+d z^{2}\right] .
\end{aligned}
$$

In this new coordinate system $(\eta, x, y, z), \eta$ denotes conformal time. Further, to solve Eq.(2), it is convenient to adopt the Coulomb gauge,

$$
\partial_{j} A^{j}=0 \quad A_{0}=0 .
$$

We can express Eq.(2) for $\mu=i$ as,

$$
A_{i}^{\prime \prime}+2 \frac{f^{\prime}}{f} A_{i}^{\prime}-a^{2} \partial_{j} \partial^{j} A_{i}=0
$$

Here prime $\left({ }^{\prime}\right)$ denotes derivative with respect to $\eta$ and $\partial^{j}$ is defined as $\partial^{j} \equiv g^{j k} \partial_{k}=a^{-2} \eta^{j k} \partial_{k}$. Promoting $A_{i}$ to an operator and imposing the quantization condition, we expand $A_{i}$ in terms of creation and annihilation operators in Fourier space [11, 22]. The evolution equation for the corresponding mode function in Fourier space $A(k, \eta)$, can be obtained from Eq.(5), and in terms of a new variable $\bar{A} \equiv a A(k, \eta)$, becomes

$$
\bar{A}^{\prime \prime}+2 \frac{f^{\prime}}{f} \bar{A}^{\prime}+k^{2} \bar{A}=0
$$

Here $k$ is the comoving wave number. We can re-express the above equation in the form of a harmonic oscillator equation with a time dependent frequency. To do this, we further define a new variable $\mathcal{A} \equiv f \bar{A}(k, \eta)$. The equation of motion in terms of this new variable is,

$$
\mathcal{A}^{\prime \prime}(k, \eta)+\left(k^{2}-\frac{f^{\prime \prime}}{f}\right) \mathcal{A}(k, \eta)=0 .
$$

Before we solve the above equation for a particular $f(\phi)$, we first define the magnetic and electric energy density respectively as,

$$
\rho_{B}=\left\langle 0\left|T_{\mu \nu}^{B} u^{\mu} u^{\nu}\right| 0\right\rangle \quad \text { and } \quad \rho_{E}=\left\langle 0\left|T_{\mu \nu}^{E} u^{\mu} u^{\nu}\right| 0\right\rangle,
$$

where $\rho_{B}$ and $\rho_{E}$ are defined as the vacuum expectation value of the respective energy momentum tensors $T_{\mu \nu}^{B}$ 
and $T_{\mu \nu}^{E}$, measured by the fundamental observers. The velocity of these observers $u^{\mu}$ is specified as $(1 / a, 0,0,0)$. In our analysis, we work with the spectral energy densities of magnetic and electric fields, $\left(d \rho_{B}(k, \eta) / d \ln k\right)$ and $\left(d \rho_{E}(k, \eta) / d \ln k\right)$. These spectral energy densities can be obtained from the Fourier transform of $\rho_{B}$ and $\rho_{E}$ in Eq.(8) [11, 22],

$$
\begin{aligned}
& \frac{d \rho_{B}(k, \eta)}{d \ln k}=\frac{1}{2 \pi^{2}} \frac{k^{5}}{a^{4}}|\mathcal{A}(k, \eta)|^{2} \\
& \frac{d \rho_{E}(k, \eta)}{d \ln k}=\frac{f^{2}}{2 \pi^{2}} \frac{k^{3}}{a^{4}}\left|\left[\frac{\mathcal{A}(k, \eta)}{f}\right]^{\prime}\right|^{2} .
\end{aligned}
$$

\section{MAGNETIC AND ELECTRIC ENERGY DENSITY DURING INFLATION}

In this analysis, we assume the background to be de Sitter during inflation. The evolution of the scale factor $a(\eta)$ with conformal time $\eta$ during de Sitter, is given by,

$$
a=\frac{-1}{H_{f} \eta}
$$

where $H_{f}=a^{\prime} / a^{2}$ is the Hubble parameter during inflation. In our normalization, $a \equiv a_{i}=1$ at $\eta_{i}=-1 / H_{f}$, where $\eta_{i}$ and $a_{i}$ are the conformal time and the scale factor at the beginning of inflation respectively. To solve Eq.(7), we need to know how $f(\phi)$ evolves with time or with expansion factor. We assume $f$ evolves as,

$$
f(a)=f_{i}\left(\frac{a}{a_{i}}\right)^{\alpha}
$$

For any model of inflation this form of $f$ can be chosen by adopting appropriate functions of $\phi[11]$. For the above mentioned form of $f(\eta)$, Eq. (7) reduces to,

$$
\mathcal{A}^{\prime \prime}(k, \eta)+\left(k^{2}-\frac{\alpha(\alpha+1)}{\eta^{2}}\right) \mathcal{A}(k, \eta)=0 .
$$

The general solution of this equation is given by,

$$
\mathcal{A}_{1}=\sqrt{-k \eta}\left[c_{1}(k) J_{-\alpha-\frac{1}{2}}(-k \eta)+c_{2}(k) J_{\alpha+\frac{1}{2}}(-k \eta)\right] .
$$

The constants $c_{1}$ and $c_{2}$ are determined by matching the solution above with the mode functions corresponding to the Bunch-Davies vacuum in the limit of $(-k \eta) \rightarrow \infty$,

$$
c_{1}=\sqrt{\frac{\pi}{4 k}} \frac{\exp \frac{i \pi \alpha}{2}}{\cos (-\pi \alpha)}, \quad c_{2}=\sqrt{\frac{\pi}{4 k}} \frac{\exp \frac{i \pi(-\alpha+1)}{2}}{\cos (-\pi \alpha)} .
$$

Using Eq.(9) and Eq.(14), the magnetic energy density spectrum in the super-horizon limit $(-k \eta)<<1$ becomes,

$$
\begin{aligned}
\frac{d \rho_{B}}{d \ln k} & \approx \frac{\mathcal{F}(n)}{2 \pi^{2}} H_{f}^{4}(-k \eta)^{4+2 n} \\
& \approx \frac{\mathcal{F}(n)}{2 \pi^{2}} H_{f}^{4}\left(\frac{k}{a H_{f}}\right)^{4+2 n}
\end{aligned}
$$

where,

$$
n=-\alpha \text { if } \alpha \geq-1 / 2 \text { and } n=1+\alpha \text { if } \alpha \leq-1 / 2
$$

and

$$
\mathcal{F}(n)=\frac{\pi}{2^{2 n+1} \Gamma^{2}(n+1 / 2) \cos ^{2}(\pi n)} .
$$

Similarly we can determine the spectral electric energy density as,

$$
\frac{d \rho_{E}}{d \ln k} \approx \frac{\mathcal{G}(m)}{2 \pi^{2}} H_{f}^{4}(-k \eta)^{4+2 m} .
$$

Here, $\mathcal{G}$ is given by,

$$
\mathcal{G}(m)=\frac{\pi}{2^{2 m+3} \Gamma^{2}(m+3 / 2) \cos ^{2}(\pi m)}
$$

and $m$ takes the values,

$$
m=-\alpha+1 \text { if } \alpha \geq 1 / 2 \text { and } m=\alpha \text { if } \alpha \leq 1 / 2 \text {. }
$$

First we explore the possibility of scale invariant magnetic field spectrum. However, we also note that scale invariance for magnetic field spectrum does not imply the scale invariance of electric field spectrum. There are two possible values of $\alpha$ for scale invariant magnetic field spectrum, namely, $\alpha=-3$ and $\alpha=2$.

For the first branch, $\alpha=-3$, we have from Eq.(20) $4+2 m=-2$. In this case, as $(-k \eta) \rightarrow 0$ (i.e. towards the end of inflation), the electric density increases rapidly as $\rho_{E} \propto(-k \eta)^{-2} \rightarrow \infty$. In this case the model runs into difficulties as the electric energy density would eventually exceed the inflaton energy density in the universe even before sufficient inflation. This problem is known as the back reaction problem. Therefore $\alpha=-3$ branch for the generation of magnetic fields is strongly constrained. Also production of large electric fields can give rise to finite conductivity due to Schwinger effect during inflation which can further stop the generation of magnetic fields as discussed in [26].

This motivates us to rather choose the second branch, $\alpha=2$, for which we have from Eq.(20) $4+2 m=2$. As $(-k \eta) \rightarrow 0, \rho_{E} \propto(-k \eta)^{2} \rightarrow 0$. This branch does not suffer from any back reaction effects, although strong coupling could potentially pose a problem here as discussed in Section V. In Section V, we also suggest a model in which strong coupling problem is avoided. Also the issue of finite conductivity arising from particle production due to Schwinger mechanism does not pose a problem for this branch. This is shown explicitly in the following section.

\section{SCHWINGER EFFECT CONSTRAINT ON MAGNETOGENESIS}

It is well known that electric fields can produce charged particles out of the vacuum due to Schwinger mechanism $[29,30]$. These particles can give rise to a finite conductivity depending on the electric field strength, resulting in 
the electric field being damped and the growth of magnetic fields being frozen [26]. Kobayashi and Afshordi [26] have determined the conductivity due to charged particles produced via Schwinger mechanism in de Sitter space-time for different strengths of electric fields. Then using the value of conductivity they have put constraints on inflationary magnetogenesis models. In our analysis we had neglected the interaction term but to check the effect of conductivity we need to reinstate the interaction term back into the equation of motion.

After including the interaction term in Eq.(5) we get,

$$
A_{i}^{\prime \prime}+\left(2 \frac{f^{\prime}}{f}+\frac{4 \pi a \sigma}{f^{2}}\right) A_{i}^{\prime}-\partial_{j} \partial_{j} A_{i}=0 .
$$

In writing this equation we have taken $j_{i}=\sigma E_{i}=$ $-\sigma(1 / a)\left(\partial A_{i} / \partial \eta\right)$ where $\sigma$ denotes conductivity due to the charged particles produced. Clearly this would reduce to the previous case (i.e. without interaction) in the limit,

$$
\left|2 \frac{f^{\prime}}{f}\right| \gg\left|\frac{4 \pi a \sigma}{f^{2}}\right|
$$

We now proceed to evaluate and examine the validity of this inequality for the growing branch, $\alpha \geq 1 / 2$. In this case Eq.(22) translates to

$$
\frac{2 \sigma \pi}{\alpha H_{f} f^{2}} \ll 1
$$

for the Schwinger effect to be unimportant. To check this bound we need to estimate $\sigma / H_{f}$, which in turn has been shown to depend on the electric field strength [26]. For the two cases $\left|e_{N} E\right| \ll H^{2}$ and $\left|e_{N} E\right| \gg H^{2}$, we adopt the results derived in [26]. We have defined $e_{N}$ as the effective charge, $e_{N}=e / f^{2}$. In the limit $f \rightarrow 1$, we get back the standard electric charge $e$. For $\left|e_{N} E\right| \gg H_{f}^{2}$

$$
\frac{\sigma}{H_{f}} \simeq \operatorname{sgn}(E) \frac{1}{12 \pi^{2}} \frac{\left|e_{N}\right|^{3} E}{H_{f}^{2}} e^{\frac{\pi m^{2}}{\left|e_{N} E\right|}}
$$

For $\left|e_{N} E\right| \ll H_{f}^{2}$, the results depend on the mass of the charged scalar field $m$. If $m>H_{f}$, we have,

$$
\frac{\sigma}{H_{f}} \simeq \frac{7}{72 \pi^{2}} \frac{e_{N}^{2} H_{f}^{2}}{m^{2}}=\frac{7}{72 \pi^{2}} \frac{e^{2} H_{f}^{2}}{f^{4} m^{2}}
$$

and if $m<H_{f}$, we have

$$
\frac{\sigma}{H_{f}} \simeq \frac{3}{4 \pi^{2}} \frac{e_{N}^{2} H_{f}^{2}}{m^{2}}=\frac{3}{4 \pi^{2}} \frac{e^{2} H_{f}^{2}}{f^{4} m^{2}} .
$$

The relations discussed above show how the conductivity behaves in the presence of electric field. It is worth emphasising that Kobayashi and Afshordi [26], have assumed that the charged scalar field and the EM field do not affect the background.
To check which one of the above cases is relevant to our analysis, we need to estimate the electric field strength in our case. Using Eq.(18),

$E^{2}(a) \equiv 8 \pi \rho_{E}=\frac{8 \pi}{f^{2}} \int d \ln k \frac{\mathcal{G}(-\alpha+1)}{2 \pi^{2}} H_{f}^{4}\left(\frac{k}{a H_{f}}\right)^{6-2 \alpha}$

From the above expression we can see that the electric energy density integral diverges for large values of $k$. We are however interested in the length scales that exit the Hubble radius during the inflationary period. This gives a range for the relevant $k$ over which the integral needs to be performed. The largest length scale of interest is the one which exits the horizon at the beginning of inflation. Hence the physical size of this scale should be equal to the Hubble radius during inflation (The Hubble radius is constant during inflation). Hence, comoving $L_{\text {upper }}=1 / H_{f} a_{i}$ which gives $k_{\text {lower }}=H_{f} a_{i}$. At a time $t$ during inflation, the scale factor $a>a_{i}$ and the comoving length exiting the horizon at that time is $L=\left(H_{f} a\right)^{-1}$ implying $k_{\text {upper }}=H_{f} a$. The smallest length scale of interest is the one which left the horizon at the end of inflation. Evaluating the integral at any time t during inflation we have, $L_{\text {lower }}=H_{f}^{-1} / a$ implying $k_{\text {upper }}=H_{f} a$. Evaluating the integral using the above limits, we have,

$$
E^{2}(a) \approx \frac{8 \pi}{f^{2}} \frac{\mathcal{G}(-\alpha+1)}{2 \pi^{2}(6-2 \alpha)} H_{f}^{4}\left(1-\left(\frac{a_{i}}{a}\right)^{6-2 \alpha}\right) .
$$

This implies,

$$
\frac{\left|e_{N} E\right|}{H_{f}^{2}} \approx \frac{2|e|}{f^{3}}\left(\frac{\mathcal{G}(-\alpha+1)}{\pi(6-2 \alpha)}\right)^{\frac{1}{2}}\left(1-\left(\frac{a_{i}}{a}\right)^{6-2 \alpha}\right)^{\frac{1}{2}} .
$$

Since $a>a_{i}$ we can neglect $a_{i} / a$ in the above expression for $1 / 2 \leq \alpha<3$. For $\alpha=3$, Eq.(29) modifies to $\left|e_{N} E\right| / H_{f}^{2}=\left(2|e| / f^{3}\right)\left(\mathcal{G}(-2) \ln \left(a / a_{i}\right) / \pi\right)^{1 / 2}$. Further assuming that $f=1$ at the beginning of inflation and that it grows during inflation, we can infer that $\left|e_{N} E\right| \ll H_{f}^{2}$ is valid throughout the inflationary regime for $1 / 2 \leq \alpha \leq 3$. As we saw for the case $\left|e_{N} E\right| \ll H_{f}^{2}$, there are two possibilities: (i) $m>H_{f}$ and (ii) $m<H_{f}$. For $m>H_{f}$, we have from Eq.(25),

$$
\frac{2 \sigma \pi}{\alpha H_{f} f^{2}} \simeq \frac{14}{72 \pi \alpha} \frac{e^{2} H_{f}^{2}}{f^{6} m^{2}}
$$

Since $f>1$ during inflation and also $m>H_{f}$ above, we can infer from the above expression that $2 \sigma \pi / \alpha H_{f} f^{2}$ is always less than 1. Hence, Eq.(23) is valid for $m>H_{f}$. This implies that magnetic field generation will not be affected in this case even if we consider the conductivity of the medium. On similar lines, for the case of $m<H_{f}$, we have from Eq.(26),

$$
\frac{2 \sigma \pi}{\alpha H_{f} f^{2}} \simeq \frac{6}{4 \alpha \pi} \frac{e^{2} H_{f}^{2}}{f^{6} m^{2}}
$$


Thus for $m<H_{f}$, even if initially $2 \sigma \pi / \alpha H_{f} f^{2} \gg 1$, as $f \propto a^{\alpha}$ grows rapidly in time, one would have $2 \sigma \pi / \alpha H_{f} f^{2} \ll 1$ and the effect of Schwinger conductivity would become negligible. By keeping in mind the validity of Eq. (23), we get the following bound for this case:

$$
H_{f}>m>\sqrt{\frac{6 e^{2}}{4 \alpha \pi}} \frac{H_{f}}{f^{3}}
$$

For $m<H_{f}$, if $m$ satisfies the above condition, conductivity will not affect inflationary magnetogenesis. This will always be satisfied as $f$ grows much larger than unity.

Thus we can conclude that our selected branch $(1 / 2 \leq$ $\alpha \leq 3$ ) is not affected by the finite conductivity of the charged particles produced due to Schwinger mechanism. The backreacting branch $\alpha=-3$ is indeed strongly constrained as already pointed out in [26].

\section{SOLVING THE STRONG COUPLING PROBLEM}

We mentioned in Section III that $\alpha=2$ branch keeps electric field under control. We also saw above that it does not face the strong constraints imposed by the Schwinger effect. However it suffers from a variant of the strong coupling problem. This problem was first pointed out by Demozzi, Mukhanov and Rubinstein [31]. It states that if $f$ grows during inflation and settles down to $f_{f}=1$ at the end of inflation, the value of $f$ would need to be very small at the beginning of inflation $\left(f=f_{i}\right)$. The effective charge defined by, $e_{N}=e / f_{i}^{2}$ would then be very high at the beginning. This would imply that the coupling between charged particles and the EM field would be unacceptably strong. Alternatively, if $f$ decreases from a large value to $f_{f}=1$, one could in principle avoid this problem. However we already pointed out that a scenario where $f$ decreases rapidly, suffers from the back reaction problem.

On the other hand, if we assume that the value of $f$ at the beginning of inflation is $f_{i}=1$, then there will be a large value of $f$ at the end of inflation and hence resulting in a very small value of coupling constant. This as such will not be a problem[22]. However, we would need to ensure that after the end of inflation, the value of $f$ decreases to its pre-inflationary value to restore back the standard couplings. This should happen within a time scale such that it does not affect the known standard physics. If this is achieved, one would have found a way of solving the strong coupling problem.

In our model, we assume that $f$ increases as a power law during de Sitter inflation beginning with a value of unity, after which it decays back to its pre-inflationary value during a matter dominated phase that lasts till reheating. The form of the power law during the inflationary phase is given by,

$$
f=f_{1} \propto a^{\alpha} \quad a_{i} \leq a \leq a_{f}
$$

and that in the post inflationary epochs by,

$$
f=f_{2} \propto a^{-\beta} \quad a_{f} \leq a \leq a_{r} .
$$

Here $a_{f}$ and $a_{r}$ denote the scale factor at the end of inflation and at the end of reheating, respectively. We consider $\alpha>1 / 2$ in our analysis and keep $\beta$ as general. Since the value of the function $f$ is taken to be unity at the beginning of inflation, its value at the end of inflation is $f_{f}=\left(a_{f} / a_{i}\right)^{\alpha}$. Further, demanding the continuity of $f$ at the end of inflation, we have,

$$
\begin{aligned}
f & =f_{1}=\left[\frac{a}{a_{i}}\right]^{\alpha} \text { for } a \leq a_{f} \\
& =f_{2}=\left[\frac{a_{f}}{a_{i}}\right]^{\alpha}\left[\frac{a}{a_{f}}\right]^{-\beta} \text { for } a_{f} \leq a \leq a_{r}
\end{aligned}
$$

In order to calculate the evolution of the vector potential after inflation, we solve Eq.(7). Since we are interested in the super-horizon scales, we express the solution (denoted by $\bar{A}_{2}$ ) as,

$$
\overline{A_{2}}=d_{1}+d_{2} \int_{\eta_{f}}^{\eta} \frac{1}{f_{2}^{2}} d \eta .
$$

Here $\eta_{f}$ is the conformal time at the end of inflation. Expressing the solution in terms of $a$, we make use of the dependence of $a$ on $\eta$ during matter dominance, $a=\left(H_{f}^{2} a_{f}^{3} / 4\right)\left(\eta+3 /\left(a_{f} H_{f}\right)\right)^{2}$. We arrive at this expression by ensuring the continuity of $a$ and $a^{\prime}$ at the end of inflation.

The solution becomes,

$$
\bar{A}_{2}=d_{1}+d_{2} \int_{a_{f}}^{a} \frac{1}{a_{f}^{2} H_{f} \sqrt{a / a_{f}} f_{2}^{2}} d a .
$$

As $\mathcal{A}=f \bar{A}$ determines the growth or decay of the magnetic and electric fields, the constant solution $\bar{A}_{2}=d_{1}$ is a decaying mode when $f$ decreases. Thus we need to have a non-zero $d_{2}$ to get growing modes during the epoch after inflation when $f$ decreases back to unity.

To find the constants $d_{1}$ and $d_{2}$, we demand that the value of $\bar{A}$ as well as its first derivative (with respect to conformal time) be continuous at the end of inflation. Using (14), the expression for $\bar{A}$ can be obtained during inflation $\left(\bar{A}_{1}\right)$ as,

$$
\begin{aligned}
\bar{A}_{1} & =\frac{\mathcal{A}_{1}}{f_{1}}=c_{1} \frac{2^{\frac{1}{2}+\alpha} k^{-\alpha} H_{f}^{\alpha}}{\Gamma\left(\frac{1}{2}-\alpha\right)}\left[1-\frac{\left(\frac{k}{a H_{f}}\right)^{2}}{4\left(\frac{1}{2}-\alpha\right)}\right. \\
& \left.+\frac{\left(\frac{k}{a H_{f}}\right)^{4}}{32\left(\frac{1}{2}-\alpha\right)\left(\frac{3}{2}-\alpha\right)}\right]+c_{2} \frac{k^{-\alpha} H_{f}^{\alpha}\left(\frac{k}{a H_{f}}\right)^{2 \alpha+1}}{2^{\alpha+\frac{1}{2}} \Gamma\left(-\frac{3}{2}+\alpha\right)} .
\end{aligned}
$$

Here we have included the higher order terms as well, that are obtained by expanding the Bessel functions in the super-horizon limit. We can see from the expression above, that the first term in the $c_{1}$ branch is time independent. If we do not consider the higher order terms in 
the $c_{1}$ branch, it would not contribute to the coefficient $d_{2}$ during derivative matching. In fact, the contribution of these higher order terms have more weightage than the $c_{2}$ branch. Matching the expression above and its derivative to the expression of $\bar{A}_{2}$ given in Eq.(38) and its derivative we get the coefficients $d_{1}$ and $d_{2}$ as,

$$
\begin{aligned}
d_{1}= & c_{1} \frac{2^{\frac{1}{2}+\alpha}\left(\frac{k}{H_{f}}\right)^{-\alpha}}{\Gamma\left(\frac{1}{2}-\alpha\right)}\left(1+\frac{\left(\frac{k}{a_{f} H_{f}}\right)^{2}}{2(2 \alpha-1)}+\frac{\left(\frac{k}{a_{f} H_{f}}\right)^{4}}{8(2 \alpha-1)(2 \alpha-3)}\right)^{\mathrm{t}} \\
& +c_{2} \frac{2^{-\frac{1}{2}-\alpha}\left(\frac{k}{H_{f}}\right)^{-\alpha}}{\Gamma\left(\frac{3}{2}+\alpha\right)}\left(\frac{k}{a_{f} H_{f}}\right)^{2 \alpha+1} \\
d_{2}= & {\left[\frac{2^{\frac{1}{2}+\alpha}\left(\frac{k}{H_{f}}\right)^{-\alpha}}{\Gamma\left(\frac{1}{2}-\alpha\right)} c_{1}\left(\frac{-k\left(\frac{k}{a_{f} H_{f}}\right)}{2 \alpha-1}+\frac{-k\left(\frac{k}{a_{f} H_{f}}\right)^{3}}{2(2 \alpha-1)(2 \alpha-3)}\right)\right.} \\
& \left.+c_{2} \frac{2^{-\frac{1}{2}-\alpha}(2 \alpha+1)(-k)\left(\frac{k}{H_{f}}\right)^{-\alpha}}{\Gamma\left(\frac{3}{2}+\alpha\right)}\left(\frac{k}{a_{f} H_{f}}\right)^{2 \alpha}\right] f_{2}^{2}\left(a_{f}\right) .
\end{aligned}
$$

After substituting $d_{1}$ and $d_{2}$ in Eq.(38), we use the solution of $\bar{A}_{2}$ in Eq.(9) and Eq.(10) for the magnetic and electric energy densities. Further retaining only the dominant terms, the post inflationary magnetic and electric energy density spectra at reheating, reduces to,

$$
\begin{aligned}
\left.\frac{d \rho_{B}(k, \eta)}{d \ln k}\right|_{R} \approx & \frac{2^{2 \alpha+1}}{32 \pi \cos ^{2}(\pi \alpha)} \frac{1}{a_{r}^{4} \Gamma^{2}\left(\frac{1}{2}-\alpha\right)} \\
& \times\left[\frac{k^{-2 \alpha+8} H_{f}^{2 \alpha-4}}{\left(\frac{1}{2}-\alpha\right)^{2} a_{f}^{4}\left(2 \beta+\frac{1}{2}\right)^{2}}\right]\left(\frac{a}{a_{f}}\right)^{4 \beta+1} \\
\left.\frac{d \rho_{E}(k, \eta)}{d \ln k}\right|_{R} \approx & \frac{2^{2 \alpha+1}}{32 \pi \cos ^{2}(\pi \alpha)} \frac{1}{a_{r}^{4} \Gamma^{2}\left(\frac{1}{2}-\alpha\right)} \\
& \times\left[\frac{k^{-2 \alpha+6} H_{f}^{2 \alpha-2} f_{2}^{4}\left(a_{f}\right)}{a_{f}^{2}\left(\frac{1}{2}-\alpha\right)^{2}}\right]
\end{aligned}
$$

If we consider $\alpha=2$ case which implies a scale invariant magnetic and electric energy density spectrum during inflation, we get a blue magnetic spectrum, with $d \rho_{B}(k, \eta) /(d \ln k) \propto k^{4}$, after inflation. Both the magnetic and electric energy density increase after inflation in this case with the electric energy density dominating over the magnetic energy density. This is evident from Fig.(1), where we show the evolution of $\rho_{B}$ (red dashed line), $\rho_{E}$ (blue dashed-dotted line) and $\rho_{\phi}$ (black solid line).

It is more convenient to express the ratios of scale factors in terms of the number of e-foldings. Let $N$ denote the number of e-foldings from the beginning to the end of inflation and $N_{r}$ denote the number of e-foldings from the end of inflation to the reheating era. Thus the ratios of scale factors become,

$$
\frac{a_{f}}{a_{i}}=e^{N} \quad \text { and } \quad \frac{a_{r}}{a_{f}}=e^{N_{r}} .
$$

By demanding $f\left(a_{r}\right)=1$, we can express $\beta=\alpha N / N_{r}$. We need to ensure that the total energy density in the electric and magnetic fields does not exceed the total energy density in the inflaton field. During inflation, this condition is always satisfied for the particular values of $\alpha$ that we consider, for which back reaction problem does not exist. The same is shown in the Fig.(1) for $\alpha=2$, the scale invariant case. The total electromagnetic energy density at the end of reheating is,

$$
\begin{aligned}
\rho_{E}+\left.\rho_{B}\right|_{R} & =\int_{a_{i} H_{f}}^{k_{r}} \frac{d \rho_{E}(k, \eta)}{d \ln k} d \ln k+\int_{a_{i} H_{f}}^{k_{r}} \frac{d \rho_{B}(k, \eta)}{d \ln k} d \ln k \\
& \approx(C+D) H_{f}^{4} e^{\alpha\left(2 N+N_{r}\right)-7 N_{r}}
\end{aligned}
$$

where $k_{i}=a_{i} H_{f}$ to $k_{f}=a_{f} H_{f}$ represents the modes which leave the horizon during inflation. Post-inflation, in the matter dominated era, a range of modes re-enter the horizon. The mode which enters the horizon at reheating is $k_{r}=a_{r} H_{r}$. Therefore, at reheating the superhorizon modes, which have been amplified have wave numbers between $k_{i}$ to $k_{r}$. In the above expression we have substituted $k_{r}=a_{r} H_{r}=a_{f} H_{f} e^{-N_{r} / 2}$. The value of $H_{r}$ is obtained by evolving the Hubble parameter during matter dominance.

The coefficients $C$ and $D$ are respectively,

$$
\begin{aligned}
& C=\frac{2^{2 \alpha+1}}{32 \pi\left(\frac{1}{2}-\alpha\right)^{2}(-2 \alpha+6) \Gamma^{2}\left(\frac{1}{2}-\alpha\right) \cos ^{2}(\alpha \pi)} \\
& D=\frac{2^{2 \alpha+1}}{32 \pi\left(\frac{1}{2}-\alpha\right)^{2}\left(2 \beta+\frac{1}{2}\right)^{2}(-2 \alpha+8) \Gamma^{2}\left(\frac{1}{2}-\alpha\right) \cos ^{2}(\alpha \pi)}
\end{aligned}
$$

The background energy density at the end of reheating is given by,

$$
\left.\rho_{\phi}\right|_{r}=g_{r} \frac{\pi^{2}}{30} T_{r}^{4} .
$$

Here $g_{r}$ represents the relativistic degrees of freedom at reheating and $T_{r}$ is the temperature at reheating. Hence by imposing the condition $\rho_{B}+\rho_{E}<\rho_{\phi}$ and substituting the value of $\rho_{B}+\rho_{E}$ from Eq.(44), we get the following constraint,

$$
2 \alpha\left(N+N_{r}\right)-(7+\alpha) N_{r}<\ln \left(\frac{\pi^{2} g_{r}}{30(C+D)}\right)-4 \ln \frac{H_{f}}{T_{r}} .
$$

In the above expression, $N$ and $N_{r}$ are not independent of $H_{f}$ and $T_{r}$. They are related by the fact that the present observable universe has to be inside the horizon at the beginning of inflation to explain the isotropy of Cosmic Microwave Background Radiation (CMB). This condition implies,

$$
\begin{aligned}
\left(a_{0} H_{0}\right)^{-1} & <\left(a_{i} H_{f}\right)^{-1} \\
\frac{1}{H_{0}} \frac{a_{r} a_{f} a_{i}}{a_{0} a_{r} a_{f}} & <\frac{1}{H_{f}} .
\end{aligned}
$$




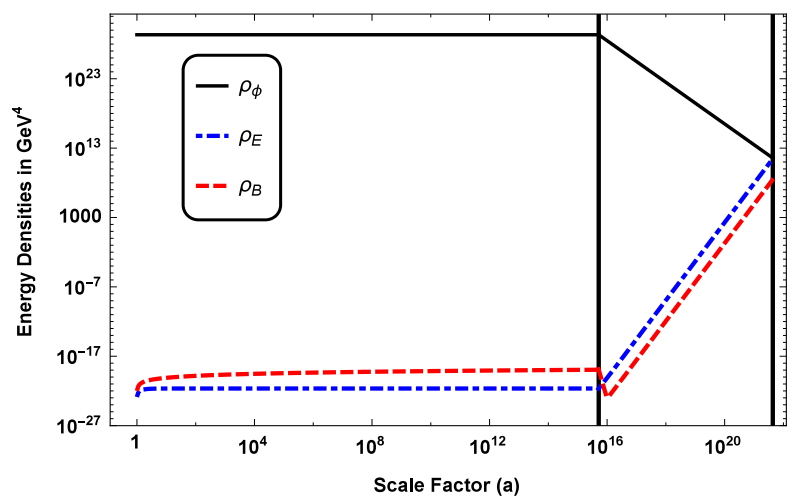

FIG. 1: In this figure evolution of $\rho_{\phi}, \rho_{E}$ and $\rho_{B}$ with scale factor has been shown. We have taken $\alpha=2$ and fixed $T_{r}$ at $100 \mathrm{GeV}$. Two vertical bold black lines represent the values of scale factor at the end of inflation $a_{f}$ and at reheating $a_{r}$ respectively. This figure shows that the energy of EM field does not overshoot the energy of the scalar field $\phi$ which decides the background geometry.

Here, $a_{0}$ and $H_{0}$ represent the scale factor and Hubble parameter today, respectively. By relating the ratios of scale factors to the number of e-folds, we get the following constraint.

$$
N+N_{r}>66.9-\ln \left(\frac{T_{r}}{H_{f}}\right)-\frac{1}{3} \ln \frac{g_{r}}{g_{0}} .
$$

To get the above expression, we have assumed radiation dominated era from reheating till today. The scale factors $a_{0}$ and $a_{r}$ are related by,

$$
\frac{a_{0}}{a_{r}}=\left(\frac{g_{r}}{g_{0}}\right)^{\frac{1}{3}} \frac{T_{r}}{T_{0}} .
$$

Here $g_{0}$ represents relativistic degree of freedom today and $T_{0}$ is the CMB temperature today.

$N_{r}$ can be written in terms of $H_{f}$ and $T_{r}$ using the inflaton energy density at the end of inflation $\left(\rho_{\text {inf }}\right)$ and reheating $\left(\left.\rho_{\phi}\right|_{r}\right)$,

$$
N_{r}=\frac{1}{3} \ln \frac{\rho_{\text {inf }}}{\left.\rho_{\phi}\right|_{r}}=\frac{1}{3} \ln \left[\frac{90 H_{f}^{2}}{8 \pi G \pi^{2} g_{r} T_{r}^{4}}\right]
$$

Substituting Eq.(48) and Eq.(50) in Eq.(46) and writing $N_{r}$ in terms of $H_{f}$ and $T_{r}$, we get the constraint

$\ln \left[\frac{C+D}{g_{r}}\left(\frac{g_{0}}{g_{r}}\right)^{\frac{2 \alpha}{3}}\left(\frac{g_{r} \pi^{2}}{30}\right)^{\frac{7+\alpha}{3}}\right]+134 \alpha+(2 \alpha+4) \ln \frac{H_{f}}{T_{r}}$

$-\frac{4(7+\alpha)}{3} \ln \left(\sqrt[4]{\frac{3 H_{f}^{2}}{8 \pi G}} \frac{1}{T_{r}}\right)<0$.

If reheating temperature and the scale of inflation satisfy the above bound, our prescribed model will be able to circumvent the strong coupling problem during inflation and back reaction in the post inflationary era.

From the inequality in Eq.(51), for a particular value of $\alpha$, once we fix $T_{r}$, we can get an upper bound on $H_{f}$ or vice-versa. After we know the above three quantities, we can proceed to calculate $N_{r}$ from Eq.(50). This value of $N_{r}$ is also an upper bound. Together with the above information, we can estimate $N$ from the bound in Eq.(48). We can further estimate the proper coherence length at reheating using the expression below,

$$
L_{c}=a_{r} \frac{\int_{0}^{k_{r}} \frac{2 \pi}{k} \frac{d \rho_{B}(k, \eta)}{d \ln k} d \ln k}{\int_{0}^{k_{r}} \frac{d \rho_{B}(k, \eta)}{d \ln k} d \ln k} .
$$

For this coherence length we can estimate the magnetic field strength at reheating using,

$$
B\left[L_{c}\right]=\left.\sqrt{8 \pi \frac{d \rho_{B}(k, \eta)}{d \ln k}}\right|_{k=\frac{2 \pi a_{r}}{L_{c}}} .
$$

\section{PREDICTED MAGNETIC FIELD STRENGTH AND COHERENCE LENGTH DUE TO FLUX FREEZING EVOLUTION}

After reheating, the universe is composed of conducting relativistic plasma. The electric fields produced in the previous epochs get shorted out. As far as the magnetic fields are concerned, several processes affect their evolution. The simplest of them is the expansion of the universe where $B \propto 1 / a^{2}$. However, on sub-Hubble scales, nonlinear processes in plasma also play an important role in their evolution. In this section we just consider the former case and the effect of nonlinear processes will be considered in the next section.

We consider reheating at different temperatures $\left(T_{r}\right)$. The lowest reheating temperature we consider is $5 \mathrm{MeV}$ as reheating below this energy scale is ruled out by Big Bang Nucleosynthesis constraints [32]. We carry out the analysis as mentioned in section $\mathrm{V}$ and estimate the coherence length $\left(L_{c}\right)$ and magnetic field strength $\left(B\left[L_{c}\right]\right)$ at reheating. To estimate the present day value of the magnetic field strength and its corresponding coherence length, we consider their evolution to be given by,

$$
\begin{gathered}
L_{c 0}=L_{c}\left(\frac{a_{0}}{a_{r}}\right), \\
B_{0}\left[L_{c 0}\right]=B\left[L_{c}\right]\left(\frac{a_{0}}{a_{r}}\right)^{-2},
\end{gathered}
$$

where $L_{c}$ and $B\left(L_{c}\right)$ are as in Eq.(52) and Eq.(53) respectively. Our analysis is repeated for a number of possible reheating temperatures, starting from $T_{r}=5 \mathrm{MeV}$ to $T_{r}=1 \mathrm{TeV}$. Of particular interest are the epochs of reheating at QCD phase transition $\left(T_{r}=150 \mathrm{MeV}\right)$ and electroweak phase transition $\left(T_{r}=100 \mathrm{GeV}\right)$. These cases have been considered for $\alpha=2$ which gives a scale invariant magnetic field spectrum during inflation. Other 
TABLE I: Present day magnetic field strength and coherence length in different models (for different values of $\alpha$ and $T_{r}$ ), assuming only flux freezing evolution.

\begin{tabular}{|l|l|l|l|l|l|l|l|}
\hline $\begin{array}{l}\text { Scale of in- } \\
\text { flation (in } \\
\mathrm{GeV})\end{array}$ & $H_{f}$ (in GeV) & $\begin{array}{l}\text { Reheating } \\
\text { Temperature } \\
T_{r}\end{array}$ & $\alpha$ & $N$ & $N_{r}$ & $\begin{array}{l}\text { Coherence } \\
\text { length } L_{c 0} \quad \text { (in } \\
\mathrm{Mpc})\end{array}$ & $\begin{array}{l}\text { Magnetic } \\
\text { field strength } \\
B_{0}\left[L_{c 0}\right](\text { in G })\end{array}$ \\
\hline $1.99 \times 10^{11}$ & $9.36 \times 10^{3}$ & $5 \mathrm{MeV}$ & 2 & 39.73 & 41.33 & $2.59 \times 10^{-5}$ & $2.04 \times 10^{-6}$ \\
\hline $4.94 \times 10^{9}$ & 5.80 & $150 \mathrm{MeV}$ & 2 & 38.41 & 31.28 & $6.45 \times 10^{-7}$ & $1.28 \times 10^{-6}$ \\
\hline $6.77 \times 10^{6}$ & $1.09 \times 10^{-5}$ & $100 \mathrm{GeV}$ & 2 & 36.18 & 13.64 & $8.84 \times 10^{-10}$ & $5.59 \times 10^{-7}$ \\
\hline $6.77 \times 10^{5}$ & $1.09 \times 10^{-7}$ & $1000 \mathrm{GeV}$ & 2 & 35.42 & 7.50 & $8.84 \times 10^{-11}$ & $3.25 \times 10^{-7}$ \\
\hline $6.33 \times 10^{2}$ & $9.51 \times 10^{-14}$ & $5 \mathrm{MeV}$ & 3 & 26.70 & 15.24 & $3.89 \times 10^{-5}$ & $2.63 \times 10^{-8}$ \\
\hline $1.03 \times 10^{2}$ & $2.52 \times 10^{-15}$ & $150 \mathrm{MeV}$ & 3 & 26.61 & 7.70 & $9.69 \times 10^{-7}$ & $9.20 \times 10^{-9}$ \\
\hline
\end{tabular}

values of $\alpha$ can also be considered if we allow a departure from scale invariance during inflation while at the same time ensuring that the backreaction problem does not arise. As an example we have considered $\alpha=3$. The latter case gives us a $d \rho_{B} / d(\ln (k)) \propto k^{2}$ magnetic spectrum instead of a $k^{4}$ spectrum for super-horizon modes in the post inflationary era. We note that for GUT scale inflation $\left(10^{14} \mathrm{GeV}\right)$, satisfying all the constraints requires a reheating temperature $\approx 10^{-8} \mathrm{GeV}$, which falls below the temperature prescribed by the BBN bound.

The results are given in Table I. As evident from the table, for a reheating temperature at $100 \mathrm{GeV}$, the magnetic field strength is $5.6 \times 10^{-7} \mathrm{G}$ at a coherence length of $8.8 \times 10^{-10} \mathrm{Mpc}$. The magnetic field strength and the

\section{NONLINEAR EVOLUTION OF MAGNETIC FIELD}

The nonlinear evolution of tangled small scale magnetic fields has been extensively discussed by Banerjee and Jedamzik [27] (see also [24]). We first summarize their arguments and then apply their results to our magnetogenesis scenarios.

Nonlinear processing becomes important when the Alfvén crossing time $\left(\eta_{N L}=\left(k V_{A}(k)\right)^{-1}\right)$ of a mode becomes smaller than the Hubble time $\left(H^{-1}\right)$. Here $V_{A}(k) \equiv \sqrt{\left(d \rho_{B} / d \ln k\right) /(\rho+p)}$ is the Alfvén velocity at $k$. The energy density and pressure of the relativistic species are denoted by $\rho$ and $p$. The Lorentz force due to the field can then drive fluid motions to the Alfvén velocity within an expansion time, provided the viscosity of the fluid is small enough. This is indeed the case in most epochs. The fluid Reynolds number $R_{e}$ is then typically large leading to cascade of energy to smaller and smaller scales or in other words a state of MHD turbulence. When such a state is achieved, energy at the coherence scale is transferred to small scales down to the dissipation scale. Since the kinetic energy comes from initial magnetic energy and there is no other energy source, magnetic energy also decays. For a blue spectrum, since coherence length, both increase, as reheating temperature decreases to $5 \mathrm{MeV}$ from $1000 \mathrm{GeV}$. Deviating from scale invariance during inflation, for $\alpha=3$, we find that at QCD phase transition $(150 \mathrm{MeV})$, the magnetic field strength achieved is $9.2 \times 10^{-9} \mathrm{G}$ at a coherence length $9.7 \times 10^{-7} \mathrm{Mpc}$. The magnetic field strength is lower than that obtained for $\alpha=2$ although the coherence length obtained is larger.

Note that in all our models, the magnetic spectrum is blue and in addition, the coherence scale also becomes smaller than the Hubble radius. Therefore it becomes necessary to consider the nonlinear processing and damping of the magnetic field, over and above its flux freezing evolution. This is taken up in the next section.

the Alfvén crossing time increases with scale $L$, the energy at the next largest scale starts dominating the spectra and this scale now becomes the new coherence length. The detailed evolution needs to be studied numerically including the effect of intervening epochs when viscosity is important. The net result of the nonlinear processing during the radiation dominated epochs can be summarized by the following evolution equations for the proper magnetic field $B^{N L}\left[L_{c}\right]$ and proper coherence length $L_{c}^{N L}$ [27],

$$
B_{0}^{N L}\left[L_{c 0}^{N L}\right]=B_{0}\left[L_{c 0}\right]\left(\frac{a_{m}}{a_{r}}\right)^{-p}, L_{c 0}^{N L}=L_{c 0}\left(\frac{a_{m}}{a_{r}}\right)^{q}
$$

where $a_{m}$ is the scale factor at radiation-matter equality, $p \equiv(n+3) /(n+5)$ and $q \equiv 2 /(n+5)$. Here, $n$ is defined in such a way that $\left(d \rho_{B} / d \ln k\right) \propto k^{n+3}$. Here we have also used the fact that the expansion factor during radiation domination varies as $a(\eta) \propto \eta$. We consider the form of evolution mentioned above up to matter-radiation equality after which $L_{c}^{N L}$ grows only logarithmically [27]. We neglect the logarithmic growth in $L_{c}^{N L}$ and evolve the two quantities till today in a similar way as mentioned in Section VI. 
The results of this calculation are shown in Table II. Compared to the previous case where nonlinear effects are not considered, we see that the coherence length is larger and the magnetic field strength is lower for the same reheating temperature. For reheating at QCD phase transition $(150 \mathrm{MeV})$, and taking $\alpha=2$ (or $n=1$ ), the magnetic field strength comes out to be $1.4 \times 10^{-12} G$ at the corresponding coherence length of $6.1 \times 10^{-4} \mathrm{Mpc}$. This value of coherence length increases for $\alpha=3$ to $2.8 \times 10^{-2} \mathrm{Mpc}$ while the corresponding magnetic field strength decreases to $3.2 \times 10^{-13} \mathrm{G}$.

TABLE II: Present day magnetic field strength and coherence length in different models (for different values of $\alpha$ and $T_{r}$ ), after taking nonlinear effects into account.

\begin{tabular}{|c|c|c|c|c|c|c|}
\hline $\begin{array}{l}\text { Scale of infla- } \\
\text { tion (in } \mathrm{GeV} \text { ) }\end{array}$ & $\begin{array}{l}\text { Reheating } \\
\text { Temperature } \\
T_{r}\end{array}$ & $\bar{\alpha}$ & $\begin{array}{l}\text { Coherence } \\
\text { length } L_{c 0}^{N L} \\
\text { (in Mpc) }\end{array}$ & $\begin{array}{l}\text { Magnetic } \\
\text { field strength } \\
B_{0}^{N L}\left[L_{c 0}^{N L}\right] \text { (in } \\
\text { G) }\end{array}$ & $\begin{array}{l}\text { Coherence } \\
\text { length } L_{c 0}^{S} \text { (in } \\
\text { Mpc) }\end{array}$ & \begin{tabular}{|l} 
Magnetic \\
field strength \\
$B_{0}^{S}\left[L_{c 0}^{S}\right]$ (in G)
\end{tabular} \\
\hline $1.99 \times 10^{11}$ & $\mathrm{MeV}$ & 2 & $6.48 \times 10^{-3}$ & $3.26 \times 10^{-11}$ & 0.102 & $5.15 \times 10^{-10}$ \\
\hline $4.94 \times 10^{9}$ & $150 \mathrm{MeV}$ & 2 & $6.09 \times 10^{-4}$ & $1.43 \times 10^{-12}$ & $1.09 \times 10^{-2}$ & $4.38 \times 10^{-11}$ \\
\hline $6.77 \times 10^{6}$ & $100 \mathrm{GeV}$ & 2 & $7.74 \times 10^{-6}$ & $7.26 \times 10^{-15}$ & $7.25 \times 10^{-4}$ & $6.81 \times 10^{-13}$ \\
\hline $6.77 \times 10^{5}$ & $1000 \mathrm{GeV}$ & 2 & $1.67 \times 10^{-6}$ & $9.11 \times 10^{-16}$ & $2.29 \times 10^{-4}$ & $1.26 \times 10^{-13}$ \\
\hline $6.33 \times 10^{2}$ & $5 \quad \mathrm{MeV}$ & 3 & 0.153 & $6.65 \times 10^{-12}$ & 0.153 & $6.65 \times 10^{-12}$ \\
\hline $1.03 \times 10^{2}$ & $150 \mathrm{MeV}$ & 3 & $2.81 \times 10^{-2}$ & $3.17 \times 10^{-13}$ & $2.81 \times 10^{-2}$ & $3.17 \times 10^{-13}$ \\
\hline
\end{tabular}

Numerical simulations by Brandenburg et. al. [33] show a slower decay of non-helical magnetic fields. This slower decay of the field is also accompanied by an apparent inverse transfer of the magnetic energy to larger scales [33-35], which is usually thought to occur only for helical fields. It would be of interest to examine the consequence of this non-helical inverse transfer to the predicted field strengths and coherence scales. The simulations of [33], start from a blue spectra with $n=2$, and show that the magnetic field energy decays as $B_{0}^{S}\left[L_{c 0}^{S}\right]=B_{0}\left[L_{c 0}\right]\left(a_{m} / a_{r}\right)^{-0.5}$ and coherence length increases as $L_{c 0}^{S}=L_{c 0}\left(a_{m} / a_{r}\right)^{0.5}$. These simulations are motivated by studying the decay of causally generated fields which typically are expected to have such a spectrum on infra-red scales. Our inflation generated fields, although having blue spectra, have more power on large scales, with $n=1$ for $\alpha=2$ case and $n=-1$ for the case when $\alpha=3$, and thus the magnetic energy could perhaps decay even more slowly. Nevertheless, to get an idea of what such a non-helical inverse transfer would imply for the field strengths and coherence scales, we adopt simply the scalings found by Brandenburg et al [33]. The results are shown in Table II for different reheating temperatures. The analysis is same as mentioned in Section VI. We note that both the coherence length and magnetic field strength are larger than the one estimated using only the standard nonlinear evolution.

For example, at a reheating temperature around QCD phase transition $(150 \mathrm{MeV})$, the coherence length increases from $6.1 \times 10^{-4} \mathrm{Mpc}$ to $1.1 \times 10^{-2}$. Further the magnetic field strength increases to $4.4 \times 10^{-11} \mathrm{G}$ from $1.4 \times 10^{-12} \mathrm{G}$. This is the case for $\alpha=2(n=1)$.
For the case of $\alpha=3(n=-1)$ the evolution relations of the magnetic field strength and the corresponding coherence length are equivalent to those obtained in [33]. Hence, the values do not change after incorporating inverse transfer. We also note that, though the magnetic field strength is larger after considering inverse transfer, it still remains far lower than the strength obtained when nonlinear effects are not taken into account. The corresponding coherence length however is considerably enhanced. We recall that for reheating at $150 \mathrm{MeV}$, the magnetic field strength without accounting for nonlinear decay, is $1.3 \times 10^{-6} \mathrm{G}$ at a coherence length of $6.5 \times 10^{-7}$ Mpc. Thus the field strength after taking account of the nonlinear decay with inverse transfer is much smaller $\left(4.4 \times 10^{-11} \mathrm{G}\right)$, and the coherence scale is much larger $\left(1.1 \times 10^{-2} \mathrm{Mpc}\right)$.

\section{CONSTRAINTS FROM $\gamma$-RAY OBERVATIONS}

We ask if the generated fields can explain the constraints from the gamma ray bounds. The $\gamma$-ray observations of $\mathrm{TeV}$ blazars suggest a lower limit on the strength of the intergalactic magnetic fields of the order of $10^{-15} \mathrm{G}$ at a comoving coherence length of $0.1 \mathrm{Mpc}$ [4]. This bound was obtained from the non-detection of secondary gamma ray emission by the Fermi telescope. The above mentioned lower limit was obtained for the case $L_{C} \gg L_{I C}$, where $L_{C}$ is the proper coherence length of the magnetic field and $L_{I C}$ is the mean free path of the charged particles that undergo inverse compton scatter- 
ing. For $L_{C} \ll L_{I C}$, the lower limit on magnetic field strength increases with coherence length as $\left(L_{c}\right)^{-1 / 2}$.

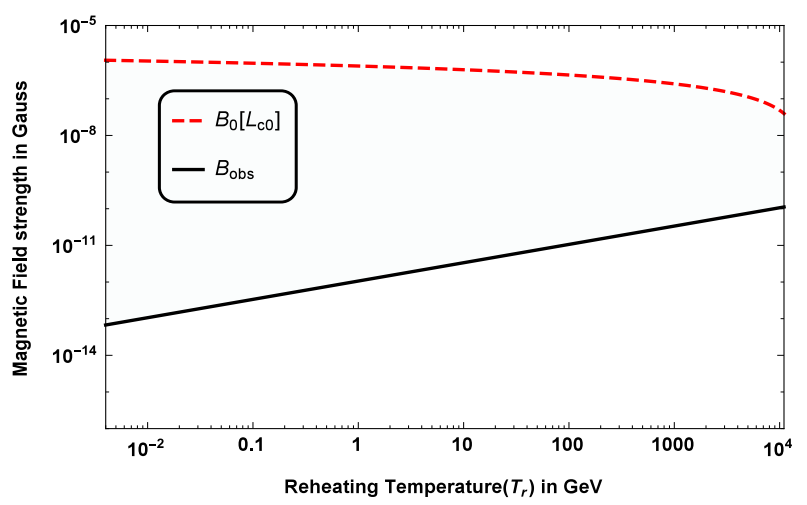

FIG. 2: This figure is for $\alpha=2$. The red dashed curve represents the maximum magnetic field strength $B_{0}\left[L_{c 0}\right]$ that can be generated in our model without taking nonlinear evolution. The black curve shows the lower bound on the observed magnetic field strength constrained by the gamma ray observations $\left(\geq 10^{-15}\right.$ at $0.1 \mathrm{Mpc}$ ) for different values of $T_{r}$. They are estimated at the coherence length of the generated magnetic field. The shaded region represents all the observationally allowed magnetic field strengths permitted by our model.

In Fig.(2) and Fig.(3) we compare the lower bound on the fields obtained from the $\gamma$-ray observations (black solid line), with the predicted field strength from our models at the coherence scale, for different reheating temperatures (red dashed line). The black line in the figures, which represents the gamma ray bound, was evaluated by scaling $10^{-15} \mathrm{G}$ at $0.1 \mathrm{Mpc}$ to the coherence length obtained for a particular reheating temperature. The scaling relation as mentioned above goes as $\left(L_{c}\right)^{-1 / 2}$. Fig.(2) is for the case when only the flux freezing evolution is taken into account (without nonlinear processing). The left panel of Fig.(3) is for the case when the standard nonlinear evolution is taken into account, while the right panel assumes the scalings implied by a possible nonlinear inverse transfer. All the figures assume $\alpha=2$, where the spectra are scale invariant during the inflationary era and transit to a blue spectrum with $n=1$ by reheating.

We can see from Fig.(2), that for the range of reheating temperatures we have considered, the magnetic field strength obtained for a particular coherence length lies well above the lower limit prescribed by the gamma ray observations. For $\alpha=2$ case, the reheating temperatures can range from a minimum value of $5 \mathrm{MeV}$ to a maximum limit of $1.7 \times 10^{4} \mathrm{GeV}$. For $\alpha=3$ case, this limit decreases to $6.7 \mathrm{GeV}$. This is because the magnetic energy density diverges during inflation and to prevent it from exceeding the inflaton energy density requires a low-scale inflation. Further to satisfy the constraint in Eq.(51), reheating temperature also has to be low.

Taking into account nonlinear evolution tightens these constraints for both $\alpha=2$ and $\alpha=3$. The change in the constraint for $\alpha=2$ case, can be seen in Fig.(3). The shaded region shown in the figure corresponds to the values of magnetic fields allowed by the gamma ray bounds. If non-helical decaying MHD turbulence does not have an inverse transfer (left panel of Fig.(3)), the reheating temperature has to be below $\approx 7 \mathrm{GeV}$ for the $\gamma$-ray bound to be satisfied. On the other hand, if one takes into account the inverse transfer as discussed in Section VII, we saw that larger magnetic field strengths are possible. Then from the right panel of Fig.(3), we see that the limits on the reheating temperatures get relaxed. In this case, the reheating temperatures allowed by the gamma ray bound increases to $\approx 4 \times 10^{3} \mathrm{GeV}$. For $\alpha=3$ case, the maximum reheating temperature is not drastically affected by considering nonlinear evolution. It decreases from $6.7 \mathrm{GeV}$ to $4 \mathrm{GeV}$. This limit is also not affected by including the inverse transfer phenomena and $T_{r}$ remains at $4 \mathrm{GeV}$. In case the lower limit on the magnetic field strength from $\gamma$-ray observations is decreased to $10^{-17} \mathrm{G}$ at $1 \mathrm{Mpc}$ as discussed in [36], our constraint on the reheating temperature will be relaxed further.

Thus we see that to resolve the strong coupling problem (by demanding $f(\phi)$ to decay to its pre-inflationary value) and also to avoid back reaction in the post inflationary era, requires both a low scale inflation and a low scale of reheating. These models however lead to magnetic field strengths and coherence scales which are consistent with the $\gamma$-ray lower limits, for reheating temperatures up to about few thousand $\mathrm{GeV}$.

We note that few earlier studies have also considered low-scale inflation in the context of inflationary magnetogenesis. Ferreira et. al. [25] have discussed a scenario wherein they have considered the back reacting branch of $f(\phi)$ during a period of low-scale inflation. Although the model successfully satisfies the gamma ray bound and avoids both strong coupling and back reaction, it violates the Schwinger effect constraint as discussed by Kobayashi and Afshordi [26]. Our model on the other hand does not run into Schwinger effect inconsistencies as discussed in Section IV.

\section{DISCUSSION AND CONCLUSIONS}

We have studied here the generation of magnetic fields during the inflationary era. As the standard Maxwell action is conformally invariant, electromagnetic (EM) field fluctuations decay with expansion as $1 / a^{2}$. Thus to generate fields of significant present day strengths, breaking of conformal invariance is imperative. One of the ways this can be done is by coupling the EM action to a function of the inflaton $\left(f^{2} F F[10]\right)$. Although such a model can lead to the generation of magnetic fields with present day strengths of interest, it suffers from several potential problems. These have been referred to as the strong coupling problem, back reaction problem and the Schwinger effect constraint.

In Section II, we have shown that in the $f^{2} F F$ model, 

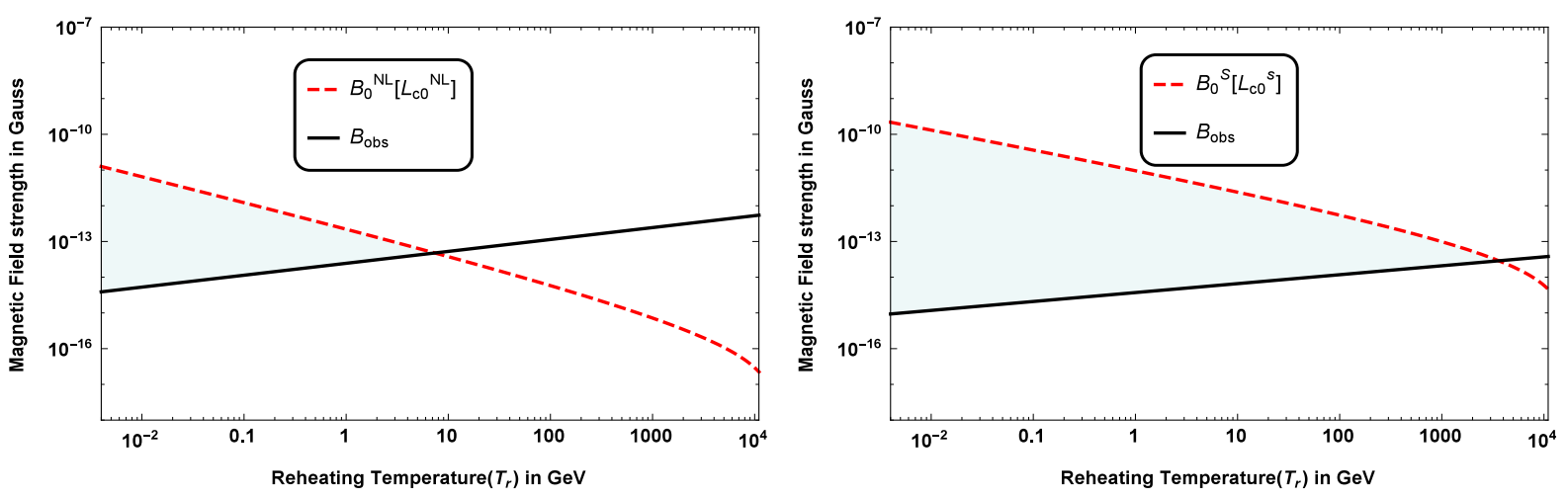

FIG. 3: These figures are for $\alpha=2$. The black curve in both the figures represents the lower bound on observed magnetic field strength constrained by the gamma ray observations $\left(\geq 10^{-15} \mathrm{G}\right.$ at $\left.0.1 \mathrm{Mpc}\right)$ for different values of $T_{r}$. They are estimated at the coherence length of the generated magnetic field. The red dashed curve in the left panel represents the maximum magnetic field strength $\left(B_{0}^{N L}\left[L_{c 0}^{N L}\right]\right)$ that can be generated in our model by taking nonlinear evolution. While the red dashed curve in the right panel represents the maximum magnetic field strength $\left(B_{0}^{S}\left[L_{c 0}^{S}\right]\right)$ that can be generated by taking direct numerical simulations results for the nonlinear evolution of magnetic fields. The shaded region in both the figures represents all the observationally allowed magnetic field strengths permitted by our model.

there are two possible evolution of $f(\phi)$ to generate scale invariant magnetic field spectrum. In the first case, $f$ increases as $a^{2}$ but if one demands that the effective electric charge is restored to the standard value at the end of inflation, the model suffers from the strong coupling problem at the beginning of inflation. In the second case, $f$ decreases as $a^{-3}$. In this case however, the resultant electric field spectrum diverges. Hence, the model suffers from the back reaction problem and Schwinger effect inconsistencies.

We have proposed a model which evades all the above mentioned problems. In our model, during inflation, $f$ increases as a power law with an exponent $\alpha$. We constrain $\alpha$ to be greater than $1 / 2$ and also such that there are no back reaction effects during inflation. The coupling function $(f)$ is assumed to begin with a standard value of unity and it increases to a large value at the end of inflation. To get back the standard coupling $(e)$, we introduce a transition at the end of inflation in the evolution of $f$. During the second part of the evolution in the post-inflationary matter dominated era, $f$ decreases as a power law with an exponent $\beta$. By demanding that the EM energy density does not back react on the background post inflation as well, we have put a bound on the reheating temperature and the scale of inflation. We note as the reheating temperature increases, the scale of inflation decreases according to the constraint in Eq.(51). Hence, the maximum reheating temperature possible is when it becomes equivalent to the scale of inflation. For the scale invariant magnetic spectrum during inflation i.e $\alpha=2$, the upper bound on the reheating temperature obtained is $\approx 10^{4} \mathrm{GeV}$.

For each reheating temperature and the scale of inflation, we have estimated the present day magnetic field strength and the corresponding coherence length. We have considered different cases in the evolution of the magnetic field after reheating. To begin with, we do not consider any nonlinear effects arising due to interaction of the magnetic modes with the plasma. For this case, we obtain fields of the order $1.3 \times 10^{-6} \mathrm{G}$ and the coherence length scales of the order $6.5 \times 10^{-7} \mathrm{Mpc}$ for a reheating temperature at the QCD epoch $(150 \mathrm{MeV})$ ( Refer to Table I). From Fig. 2, we see that for all reheating temperatures below about $10^{4} \mathrm{GeV}$, the magnetic field strengths and coherence scales are large enough to satisfy the gamma ray bound.

Taking nonlinear evolution and the resulting turbulent decay into account, we find that the $\gamma$-ray observations lead to an upper bound on the reheating temperature of about $7 \mathrm{GeV}$ (the left panel of Fig (3)). A model which does satisfy the $\gamma$-ray constraint, is when reheating occurs at the QCD epoch. The coherence length is enhanced to $6.1 \times 10^{-4} \mathrm{Mpc}$ and the magnetic field strength is decreased to about $1.4 \times 10^{-12} \mathrm{G}$.

However there is also the phenomena of inverse transfer [33] (predicted by numerical simulations) of non-helical MHD turbulence which needs to be taken into account. When this is also incorporated in our calculations, we see an improvement in the constraint on reheating temperatures and strength of the magnetic fields. The upper bound on reheating temperature increases to $\approx 4 \times 10^{3}$ $\mathrm{GeV}$. For a reheating temperature at $100 \mathrm{GeV}$, the coherence length is enhanced to $7.3 \times 10^{-4} \mathrm{Mpc}$ from $8.8 \times 10^{-10} \mathrm{Mpc}$ (which is obtained if we assume only pure flux freezing evolution). On the other hand the magnetic field strength at the above mentioned coherence scales decreases to $6.8 \times 10^{-13} \mathrm{G}$ from $5.6 \times 10^{-7} \mathrm{G}$.

One possible scenario whereby the coupling function $f$ transits from a growing function to one that decays back to unity can perhaps be realized in models of hybrid in- 
flation [37]. In hybrid inflation, two interacting scalar fields, $\phi$ and $\sigma$, are employed. During inflation, the inflaton field $(\phi)$ slow rolls and the other field $(\sigma)$ is static. To end inflation, $\phi$ triggers $\sigma$ to rapidly roll down the potential. The transition in $f$ can be explained by making $f$ a function of both these scalar fields. The function $f$ could increase as $\phi$ slow rolls during inflation but at the end of inflation, it shifts to a function of $\sigma$. It is brought down to its initial value as $\sigma$ cascades down. More work is needed to explore this idea further.

In our analysis, we have only looked at the generation of non-helical magnetic fields. Helical magnetic fields on the other hand will provide a much bigger advantage since inverse cascade leads to an even milder decrease in the strength of the magnetic fields while increasing the coherence lengths of the fields. We intend to look at this case in the future. There is also the question of grav- itational waves production from anisotropic stresses of these generated magnetic fields as discussed by Caprini and Durrer [38]. It would be of interest to study gravitational wave generation in our model and check whether they could be detected in future generation of detectors and thus probe magnetogenesis.

\section{ACKNOWLEDGMENTS}

RS, SJ and TRS acknowledge the facilities at IRC, University of Delhi as well as the hospitality and resources provided by IUCAA, Pune where part of this work was carried out. The research of RS is supported by SRF from CSIR, India under grant 09/045(1343)/2014-EMRI. The research of SJ is supported by UGC Non-NET fellowship, India. TRS acknowledges the Project grant from SERB EMR/2016/002286.
[1] R. Beck, Space Sci. Rev. 99, 243 (2001), arXiv:astro-ph/0012402 [astro-ph].

[2] T. E. Clarke, P. P. Kronberg, and H. Boehringer, Astrophys. J. 547, L111 (2001), arXiv:astro-ph/0011281 [astro-ph].

[3] L. M. Widrow, Reviews of Modern Physics 74, 775 (2002) astro-ph/0207240.

[4] A. Neronov and I. Vovk, Science 328, 73 (2010), arXiv:1006.3504 [astro-ph.HE].

[5] I. B. Zeldovich, A. A. Ruzmaikin, and D. D. Sokolov, eds., New York, Gordon and Breach Science Publishers (The Fluid Mechanics of Astrophysics and Geophysics. Volume 3), 1983, 381 p. Translation., Vol. 3 (1983).

[6] A. Shukurov, in Mathematical Aspects of Natural Dynamos, edited by E. Dormy and A. M. Soward (Chapman \& Hall/CRC, 2007) pp. 313-359, arXiv:astro-ph/0411739.

[7] A. Brandenburg and K. Subramanian, Phys. Rep. 417, 1 (2005), astro-ph/0405052.

[8] R. M. Kulsrud and E. G. Zweibel, Reports on Progress in Physics 71, 046901 (2008), arXiv:0707.2783.

[9] M. S. Turner and L. M. Widrow, Phys. Rev. D 37, 2743 (1988).

[10] B. Ratra, Astrophys. J. 391, L1 (1992).

[11] J. Martin and J. Yokoyama, Journal of Cosmology and Astroparticle Physics 1, 025 (200 arXiv:0711.4307.
[12] T. Kobayashi, Journal of Cosmology and Astroparticle Physic

[13] K. Atmjeet, I. Pahwa, T. R. Seshadri, and K. Subramanian, Phys. Rev. D 89, 063002 (2014), arXiv:1312.5815 [astro-ph.CO].

14] L. Campanelli, The European Physical Journal C 75, 278 (2015).arXiv:0907.1030 [astro-ph.CO].

[15] G. Tasinato, Journal of Cosmology and Astroparticle Physics[20115., 040. (20155). Salas, M. Lattanzi, G. Mangano,

[16] T. Vachaspati, Physics Letters B 265, 258 (1991).

[17] G. Sigl, A. V. Olinto, and K. Jedamzik, Phys. Rev. D55, 4582 (1997), arXiv:astro-ph/9610201 [astro-ph]. 20144y,s048)e(201D496, 025012 (2017), arXiv:1704.05076 [gr-qc].

[31] V. Demozzi, V. Mukhanov, and H. Rubinstein, G. Miele, S. Pastor, and O. Pisanti, Phys. Rev. D 92, 123534 (2015), arXiv:1511.00672.

[33] A. Brandenburg, T. Kahniashvili, and A. G. Tevzadze, Physical Review Letters 114, 075001 (2015),
[18] L. S. Kisslinger, Phys. Rev. D 68, 043516 (2003), hep-ph/0212206.

[19] A. G. Tevzadze, L. Kisslinger, A. Brandenburg, and T. Kahniashvili, Astrophys. J. 759, 54 (2012), arXiv:1207.0751 [astro-ph.CO].

[20] D. Grasso and H. R. Rubinstein, Phys. Rep. 348, 163 (2001), astro-ph/0009061.

[21] R. Durrer and A. Neronov, Astron. Astrophys. Rev. 21, 62 (2013), arXiv:1303.7121 [astro-ph.CO].

[22] K. Subramanian, Astronomische Nachrichten 331, 110 (2010), arXiv:0911.4771.

[23] A. Kandus, K. E. Kunze, and C. G. Tsagas, Phys. Rept. 505, 1 (2011), arXiv:1007.3891 [astro-ph.CO].

[24] K. Subramanian, Reports on Progress in Physics 79, 076901 (2016), arXiv:1504.02311.

[25] R. J. Z. Ferreira, R. K. Jain, and M. S. Sloth, Journal of Cosmology and Astroparticle Physics 10, 004 (2013), arXiv:1305.7151 [astro-ph.CO].

[26] T. Kobayashi and N. Afshordi, Journal of High Energy Physics 10, 166 (2014), arXiv:1408.4141 [hep-th].

[27] R. Banerjee and K. Jedamzik, Phys. Rev. D 70, 123003 (2004), astro-ph/0410032.

[28] L. Parker, Physical Review Letters 21, 562 (1968).

J. S. Schwinger, Phys. Rev. 82, 664 (1951).

[30] R. Sharma and S. Singh, Journal of Cosmology and Astroparticle Physics 8, 025 (2009), arXiv:1404.2238. 
[34] A. Brandenburg and T. Kahniashvili, Physical Review Letters 118, 055102 (2017), arXiv:1607.01360 [physics.flu-dyn].

[35] J. Zrake, Astrophys. J. 794, L26 (2014), arXiv:1407.5626 [astro-ph.HE].
[36] A. M. Taylor, I. Vovk, and A. Neronov, Astron. Astrophys. 529, A144 (2011), arXiv:1101.0932 [astro-ph.HE].

[37] A. Linde, Phys. Rev. D 49, 748 (1994), astro-ph/9307002.

[38] C. Caprini and R. Durrer, Phys. Rev. D 65, 023517 (2001), astro-ph/0106244. 